\title{
Co-analysis of Maps of Atrophy Rate and Atrophy State in Neurodegeneration
}

\author{
Valerie A. Cardenas and Colin Studholme \\ Department of Radiology, University of California, San Francisco and San Francisco \\ VA Medical Center
}

\begin{abstract}
The variability in atrophy rate in patients with neurodegenerative disease can be partially explained by disease stage. Since atrophy state (baseline scan tissue volume) correlates with cognitive impairment and disease stage, we use local atrophy state as a covariate in the analysis of local atrophy rate. A general linear model with a spatially varying dependent variable was employed to study the effects of disease over the brain. An efficient method of solving this spatially varying model was developed, implemented, and applied to maps of atrophy state and rate derived from serial MRI data of 13 Semantic Dementia (SD) and 13 control subjects. Our results demonstrate that group differences in atrophy rate are affected by the inclusion of atrophy state in the model. Specifically, estimated contrasts in atrophy rate between controls and SD in the left temporal lobe white matter increased when the local rate was covaried for atrophy state.
\end{abstract}

\section{Introduction}

Spatial morphometric techniques such as voxel based morphometry (VBM) [12] and deformation tensor morphometry [3] have been used to explore separately both the patterns of atrophy state (amount of tissue lost) in neurodegenerative disease and the atrophy rate (rate of tissue loss). These methods have added significantly to the understanding of the progression of degenerative disease in different regions of the brain.

From both manual region based measurements and spatial morphometric approaches, significant brain atrophy in various anatomical structures has been reported cross sectionally in patients compared to controls [567. Many methods also exist for estimating atrophy rate from longitudinal structural images 8910. These show more diagnostic promise than cross sectional tissue volume measurements from a single subject because they remove the underlying variability in local tissue volume and focus simply on the change. There is evidence that the rate of loss may increase with the progression of a disease [11]12], but it is not clear how this relationship may vary for different regions in the brain. Large clinical studies may include patients at a range of stages of the disease. However, staging of a subject commonly depends only on imprecise clinical neurological testing. True differences in atrophy state and rate between patients and controls may therefore be obscured if the stage of the disease is poorly defined, 
as illustrated by Fig 1 In this paper we therefore explore the use of local atrophy state as a covariate in the analysis of local atrophy rate, since atrophy state has been shown to be correlated with degree of cognitive impairment [13].
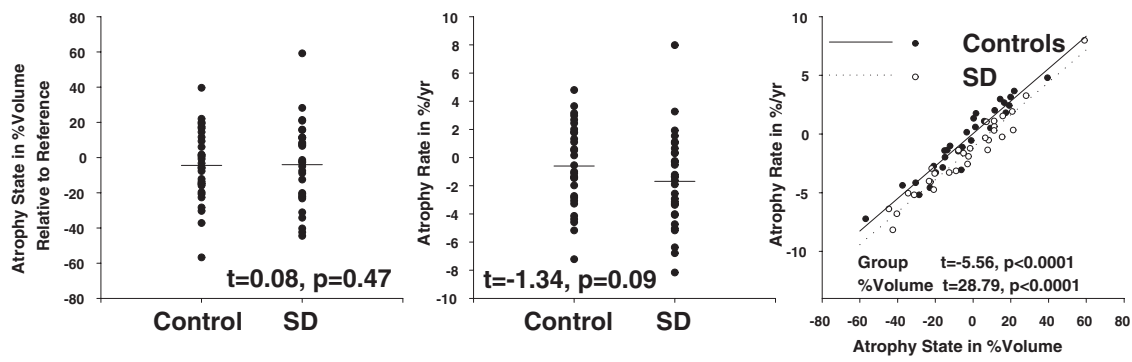

Fig. 1. On the left is a scatterplot of simulated atrophy states for controls and Semantic Dementia (SD) patients, showing a small, not significant difference in group means $(p=$ 0.47). In the middle is a scatterplot of simulated atrophy rates, where the difference in rates nearly reaches statistical significance $(p=0.09)$. The right scatterplot shows atrophy state vs. atrophy rate. When the group comparison of atrophy rate is covaried for atrophy state, the group difference is highly significant $(p<0.0001)$

Spatial Morphometric techniques such as deformation morphometry quantify differences between brains without a priori definition of ROIs, by fitting a fixed, common linear model to the deformation parameters and computing statistics at the voxel level. Covarying for atrophy state is complicated because the atrophy state varies spatially, so the linear model cannot be fixed. In this paper, we describe a linear model with a spatially varying dependent variable and present a technique for efficient solution of the model, and show the results of applying the model to patients with Semantic Dementia (SD).

\section{Methods}

\subsection{Linear Model}

We are going to use a general linear model [14[15] to analyze our maps of atrophy rate. Given $n$ images that have been registered to a common coordinate system, we fit the following linear model at each voxel $i$ in the image,

$$
\mathbf{y}\left(\mathbf{v}_{i}\right)=\mathbf{A x}\left(\mathbf{v}_{i}\right),
$$

where $\mathbf{v}_{i}=\left(v_{i 1}, v_{i 2}, v_{i 3}\right)$ denotes the location of voxel $i$ in standard space, $\mathbf{y}\left(\mathbf{v}_{i}\right)$ is an $n$-vector of measurements (e.g., gray matter density, relative local contraction 
or expansion) at voxel $\mathbf{v}_{i}, \mathbf{x}\left(\mathbf{v}_{i}\right)$ is a $p$-vector of parameters (e.g., effect of disease, effect of age) to be estimated at voxel $\mathbf{v}_{i}$, and $\mathbf{A}$ is an $n \times p$ design matrix, where the $p$ columns of $\mathbf{A}$ contain categorical or continuous variables for $n$ subjects (e.g., column of subject diagnosis variables, column of subject ages). In this model, the independent variables that compose $\mathbf{A}$ are not dependent on spatial location within the image. For the usual case when $n>p$ and we have more equations than unknowns, we have a linear least-squares problem and can solve the reduced set of equations,

$$
\left(\mathbf{A}^{T} \mathbf{A}\right) \mathbf{x}\left(\mathbf{v}_{i}\right)=\mathbf{A}^{T} \mathbf{y}\left(\mathbf{v}_{i}\right)
$$

One method of solving these equations is to compute the Cholesky decomposition $\mathbf{L L}^{T}$ of $\mathbf{A}^{\mathbf{T}} \mathbf{A}$, where $\mathbf{L}$ is lower triangular. The elements $L_{k j}$ of $\mathbf{L}$ are computed from the elements $A_{k j}$ of $\mathbf{A}$ as follows,

$$
\begin{gathered}
L_{k k}=\sqrt{A_{k k}-\sum_{m=1}^{k-1} L_{m k}^{2}} \text { for } k=j, \\
L_{k j}=\left(A_{k j}-\sum_{m=1}^{j-1} L_{k m} L_{j m}\right) / L_{j j} \quad \text { for } k>j,
\end{gathered}
$$

where $k$ indexes rows and $j$ indexes columns. We can then solve the trivial triangular systems $\mathbf{L b}\left(\mathbf{v}_{i}\right)=\mathbf{A}^{T} \mathbf{y}\left(\mathbf{v}_{i}\right)$ and $\mathbf{b}\left(\mathbf{v}_{i}\right)=\mathbf{L}^{T} \mathbf{x}\left(\mathbf{v}_{i}\right)$. Because the design matrix $\mathbf{A}$ is fixed over the image, the Cholesky decomposition of $\mathbf{A}^{\mathbf{T}} \mathbf{A}$ can be computed once, and parameter estimates $\mathbf{x}\left(\mathbf{v}_{i}\right)$ can be easily solved at each voxel by substituting a new $\mathbf{y}\left(\mathbf{v}_{i}\right)$.

The estimation of t-statistics for the parameter estimates requires the computation of the diagonal entries $u_{j j}$ of $\left(\mathbf{A}^{\mathbf{T}} \mathbf{A}\right)^{-1}$, where $j$ indexes columns. Because the inverse of a lower triangular matrix can be easily computed row by row, computation of $u_{j j}$ is facilitated by using $\left(\mathbf{A}^{T} \mathbf{A}\right)^{-1}=\left(\mathbf{L}^{-1}\right)^{T} \mathbf{L}^{-1}$, then recognizing that $u_{j j}=\mathbf{l}_{j} \cdot \mathbf{l}_{j}$ where $\mathbf{l}_{j}$ are the columns of $\mathbf{L}^{-1}$.

If we want to compare the rate of atrophy between groups, while covarying for the spatially varying tissue volume at baseline, the linear model then becomes,

$$
\mathbf{y}\left(\mathbf{v}_{i}\right)=\mathbf{A}\left(\mathbf{v}_{i}\right) \mathbf{x}\left(\mathbf{v}_{i}\right)
$$

where one column of the design matrix $\mathbf{A}\left(\mathbf{v}_{i}\right)$ is the tissue volume at baseline for voxel $\mathbf{v}_{i}$. Even though all other columns of $\mathbf{A}\left(\mathbf{v}_{i}\right)$ are fixed (e.g., diagnosis and age independent variables), the design matrix is no longer fixed over the image due to the inclusion of baseline tissue volumes at voxel $\mathbf{v}_{i}$, and subsequently the Cholesky decomposition will change at every voxel in the image. However, by choosing to make the tissue volume at voxel $\mathbf{v}_{i}$ the $p^{\text {th }}$ column of $\mathbf{A}\left(\mathbf{v}_{i}\right)$, we can avoid computing the full Cholesky decomposition of $\mathbf{A}\left(\mathbf{v}_{i}\right)^{T} \mathbf{A}\left(\mathbf{v}_{i}\right)$. Let $\mathbf{c}_{j}$ denote the $j^{\text {th }}$ column of $\mathbf{A}\left(\mathbf{v}_{i}\right)$, where only the last column $\mathbf{c}_{p}\left(\mathbf{v}_{i}\right)$ varies spatially, then 


$$
\mathbf{A}\left(\mathbf{v}_{i}\right)^{T} \mathbf{A}\left(\mathbf{v}_{i}\right)=\left[\begin{array}{cccc}
\mathbf{c}_{1} \cdot \mathbf{c}_{1} & \mathbf{c}_{1} \cdot \mathbf{c}_{2} & \cdots & \mathbf{c}_{1} \cdot \mathbf{c}_{p}\left(\mathbf{v}_{i}\right) \\
\mathbf{c}_{1} \cdot \mathbf{c}_{2} & \mathbf{c}_{2} \cdot \mathbf{c}_{2} & \cdots & \mathbf{c}_{2} \cdot \mathbf{c}_{p}\left(\mathbf{v}_{i}\right) \\
\vdots & \vdots & \ddots & \vdots \\
\mathbf{c}_{1} \cdot \mathbf{c}_{p}\left(\mathbf{v}_{i}\right) & \mathbf{c}_{2} \cdot \mathbf{c}_{p}\left(\mathbf{v}_{i}\right) \cdots & \mathbf{c}_{p} \cdot \mathbf{c}_{p}\left(\mathbf{v}_{i}\right)
\end{array}\right]
$$

where $\mathbf{c}_{i} \cdot \mathbf{c}_{j}$ denotes the dot product of vectors $\mathbf{c}_{i}$ and $\mathbf{c}_{j}$. As shown in (6), only the last row and column of $\mathbf{A}\left(\mathbf{v}_{i}\right)^{T} \mathbf{A}\left(\mathbf{v}_{i}\right)$ change at each voxel, by Eq. (34) only the last row of the Cholesky factor $\mathbf{L}\left(\mathbf{v}_{i}\right)$ changes at each voxel, and in turn only the last row of $\mathbf{L}\left(\mathbf{v}_{i}\right)^{-1}$ changes at each voxel. Because of this, we can compute $\mathbf{L}\left(\mathbf{v}_{1}\right)$, then for each voxel update the last row of $\mathbf{L}\left(\mathbf{v}_{i}\right)$ using the last row of $\mathbf{A}\left(\mathbf{v}_{i}\right)^{T} \mathbf{A}\left(\mathbf{v}_{i}\right)$ and the previously computed $\mathbf{L}\left(\mathbf{v}_{1}\right)_{k j}$. Since the computational cost of the Cholesky decomposition is $p^{3} / 3$ flops, computing only the last row of $\mathbf{L}\left(\mathbf{v}_{i}\right)$ saves $(p-1)^{3} / 3$ flops at each voxel. We can also compute $\mathbf{L}\left(\mathbf{v}_{1}\right)^{-1}$, then simply update the last row of $\mathbf{L}\left(\mathbf{v}_{i}\right)^{-1}$ using the last row of $\mathbf{L}\left(\mathbf{v}_{i}\right)$ and the previously computed entries of $\mathbf{L}\left(\mathbf{v}_{1}\right)^{-1}$. Since the computational cost of computing a full inverse is $p^{3}$, computing only the last row of $\mathbf{L}\left(\mathbf{v}_{i}\right)^{-1}$ saves nearly $(p-1)^{3}$ flops. Computing only the last row of $\mathbf{A}\left(\mathbf{v}_{i}\right)^{T} \mathbf{A}\left(\mathbf{v}_{i}\right)$ provides an additional savings of about $(p-1)^{2} n$ flops, which may actually be a greater savings for a large number of subjects $n$ and small number of parameters $p$. For a typical analysis with approximatly 1.5 million voxels, our implementation saves about $1.5 \times 10^{6}\left(p^{3}+(p-1)^{2} n\right)$ flops.

\subsection{Application to Semantic Dementia}

Subjects. Thirteen subjects ( $62 \pm 7$ yrs, 8 men) diagnosed with Semantic Dementia (SD) and 13 cognitively normal age and sex matched controls $(64 \pm 9$ yrs, 8 men) were studied at least twice with a 3D gradient echo T1 weighted anatomical MPRAGE sequence $(\mathrm{TR}=10 \mathrm{~ms}, \mathrm{TE}=4 \mathrm{~ms})$. The images were reconstructed with a coronal slice plane at a voxel size of $1 \times 1 \times 1.5 \mathrm{~mm}^{3}$. The interval between images was $2.9 \pm 1.2$ yrs for controls and $1.0 \pm 0.2$ yrs for SD. These individuals were selected from a larger group of patients participating in research at the San Francisco VA hospital and the UCSF Memory and Aging Center.

Maps of Atrophy State and Rate. The scan from a single 72 year old cognitively normal female was used as the reference for this study, rather than a group average, in order to retain the finest anatomical structures in the target for accurate registration. An entropy driven B-Spline Free Form deformation algorithm [16,17] was used to register individual scans to the reference atlas. The Jacobian determinant of this transformation, giving the fractional volume contraction or expansion relative to the reference, was used to characterize the atrophy state $s\left(\mathbf{v}_{i}\right)$ at each voxel $\mathbf{v}_{i}$. Because both global (due to headsize differences between reference and subject) and local tissue changes are included in the estimate $s\left(\mathbf{v}_{i}\right)$, we computed the average atrophy state over the intracranial vault $V$ as $\frac{1}{V} \sum_{i=1}^{V} s\left(\mathbf{v}_{i}\right)$, and we divided $s\left(\mathbf{v}_{i}\right)$ by this value to create a normalized atrophy state $s^{\prime}\left(\mathbf{v}_{i}\right)$ that reflected only local changes relative to headsize. Detailed method descriptions are found in 18,19 . 
At higher resolution, the same general approach used to create maps of atrophy state can be used to register and model spatial changes within subject between multiple scans 20. The previously estimated transformation between the initial time point of each subject and the reference anatomy then allows the spatial normalization of annualized pointwise volume change for each subject, yielding atrophy rate estimates $r\left(\mathbf{v}_{i}\right)$ at each voxel $\mathbf{v}_{i}$ in standard space.

Model of Atrophy Rate in Semantic Dementia. Linear models were fit to each voxel and t-statistics calculated to locate points where voxel level differences in the annualized rate of atrophy occurred. The effect of disease was initially examined using an ANCOVA analysis at each voxel with diagnosis (SD vs. control) as a categorical predictor while covarying for age. This is shown by

$$
\mathbf{r}\left(\mathbf{v}_{i}\right)=x\left(\mathbf{v}_{i}\right)_{1} \iota+x\left(\mathbf{v}_{i}\right)_{2} \mathbf{d}+x\left(\mathbf{v}_{i}\right)_{3} \mathbf{a},
$$

where $\mathbf{r}\left(\mathbf{v}_{i}\right)$ is an vector of atrophy rates at voxel $\mathbf{v}_{i}$ from $n$ subjects, $x\left(\mathbf{v}_{i}\right)_{j}$ are elements of the parameter vector $\mathbf{x}\left(\mathbf{v}_{i}\right), \boldsymbol{\iota}$ is an $n$-vector of ones to model an intercept, and $\mathbf{d}$ and $\mathbf{a}$ are $n$-vectors of subject diagnoses and ages. The effect of disease was then examined using a modified ANCOVA analysis, where atrophy state normalized by headsize was included as a covariate, as shown by

$$
\mathbf{r}\left(\mathbf{v}_{i}\right)=x\left(\mathbf{v}_{i}\right)_{1} \iota+x\left(\mathbf{v}_{i}\right)_{2} \mathbf{d}+x\left(\mathbf{v}_{i}\right)_{3} \mathbf{a}+x\left(\mathbf{v}_{i}\right)_{4} \mathbf{s}^{\prime}\left(\mathbf{v}_{i}\right),
$$

where $\mathbf{s}^{\prime}\left(\mathbf{v}_{i}\right)$ is an $n$-vector of normalized atrophy state at voxel $\mathbf{v}_{i}$. For our analysis of 13 controls (coded 0) and $13 \mathrm{SD}$ (coded 1), (8) can be written in matrix notation (as in (5))

$$
\left[\begin{array}{c}
r\left(\mathbf{v}_{i}\right)_{1} \\
\vdots \\
r\left(\mathbf{v}_{i}\right)_{13} \\
r\left(\mathbf{v}_{i}\right)_{14} \\
\vdots \\
r\left(\mathbf{v}_{i}\right)_{26}
\end{array}\right]=\left[\begin{array}{cccc}
1 & 0 & 61 & s^{\prime}\left(\mathbf{v}_{i}\right)_{1} \\
\vdots & \vdots & \vdots & \vdots \\
1 & 0 & 68 & s^{\prime}\left(\mathbf{v}_{i}\right)_{13} \\
1 & 1 & 68 & s^{\prime}\left(\mathbf{v}_{i}\right)_{14} \\
\vdots & \vdots & \vdots & \vdots \\
1 & 1 & 58 & s^{\prime}\left(\mathbf{v}_{i}\right)_{26}
\end{array}\right]\left[\begin{array}{c}
x\left(\mathbf{v}_{i}\right)_{1} \\
x\left(\mathbf{v}_{i}\right)_{2} \\
x\left(\mathbf{v}_{i}\right)_{3} \\
x\left(\mathbf{v}_{i}\right)_{4}
\end{array}\right]
$$

\section{Results}

Fig 2 illustrates how covarying for atrophy state affects the estimation of atrophy rate differences between controls and SD. The middle panel of Fig 2 is the map of estimated $x\left(\mathbf{v}_{i}\right)_{2}$ from Eq7. The bottom panel is the same map when atrophy state is included as a covariate (see Eq 8). Although both regions are statistically very significant $\left(p<5.0 \times 10^{-6}\right)$, the white matter of the left anterior temporal lobe is estimated to atrophy at a faster rate (white in bottom panel of Fig 2) in $\mathrm{SD}$ compared to controls when atrophy state is considered. This suggests that the rate of white matter loss in the anterior temporal lobe varies among our SD patients, and that the rate of white matter loss is related to white matter volume 
in the baseline scan. When only group differences are modeled, the variability in rates of white matter atrophy lead to a smaller group estimate (in the illustrated region, SDs atrophy about $1.57 \%$ yr faster than controls). When atrophy state is included, the variability in rates of white matter atrophy are partially explained by white matter volume in the first scan, and a larger estimate of the group effect is obtained $(1.84 \% / \mathrm{yr})$.

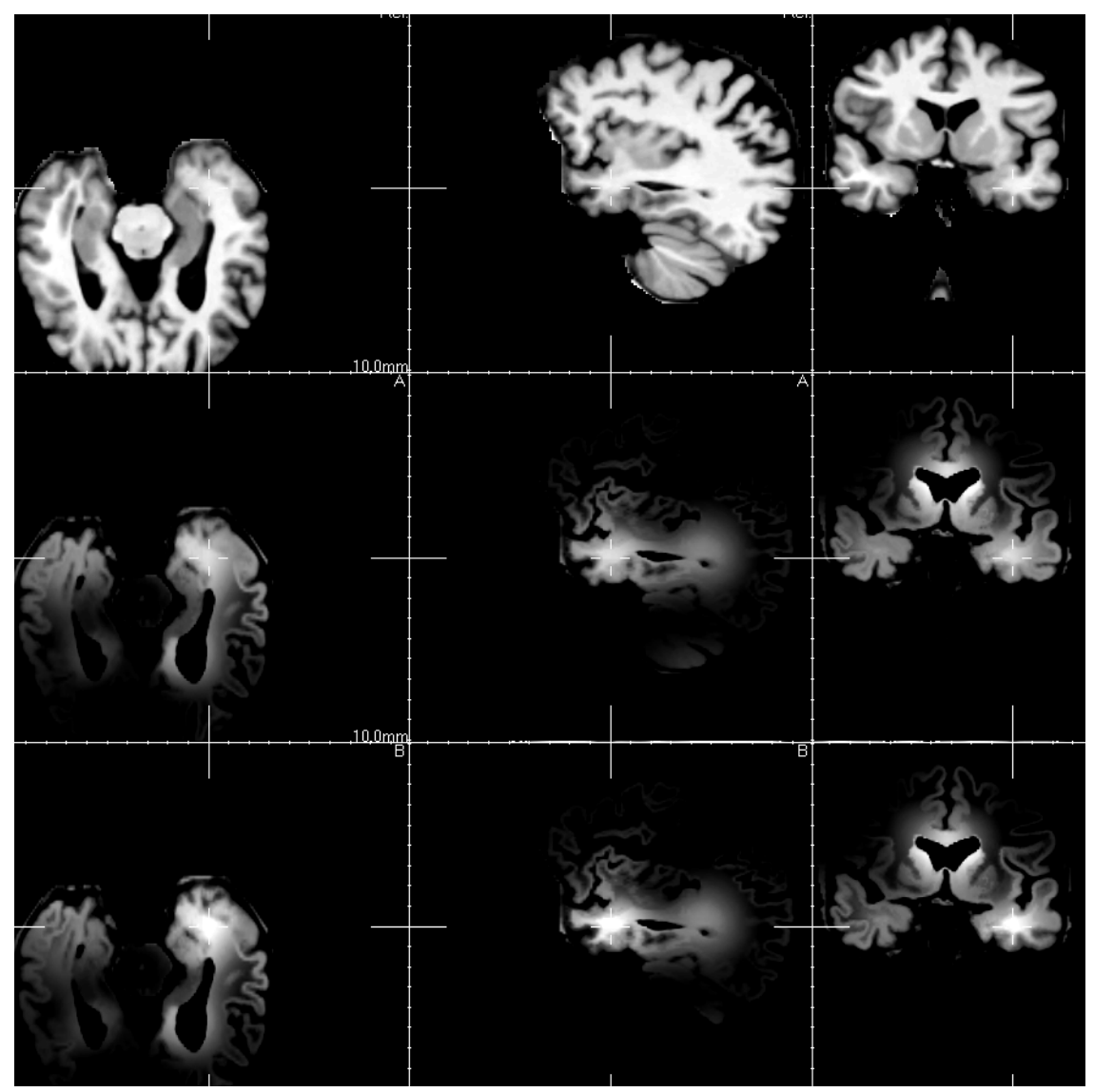

Fig. 2. Differences in atrophy rate between SD and controls when atrophy state is not included (middle panel) and is included (bottom panel) in the model, shown as a grey scale between $0.2 \%$ and $2 \%$ per year. Top row shows average MRI of the group after spatial normalization. 


\section{Discussion}

The inclusion of atrophy state as a spatially varying independent variable in our general linear model resulted in the estimation of a larger group effect on the atrophy rate of white matter in the anterior temporal lobe. Including atrophy state had little effect on the estimation of group differences in the cortex or ventricular CSF, suggesting that the relationship between atrophy rate and disease stage varies spatially. Although our results suggest that there is a stronger association between cognition and rate of white matter atrophy than rate of cortical atrophy, our cohort of $13 \mathrm{SD}$ patients may have simply been too few to adequately model the relationship in the cortex. We have shown the feasibility of the co-analysis of maps of atrophy rate and atrophy state, and demonstrated its importance using images from 13 SD patients and 13 controls. Future work will include expanding our SD group, examining other neurodegenerative diseases, and investigating permutation testing to correct for multiple comparisons.

Acknowledgements. This work was funded by a Whitaker foundation grant (RG-01-0115) and NIH grant MH65392-01. Image data used in this work was acquired as part of the NIH funded grants AG12435, AG10897, P01AG19724 and P01AA11493. The authors wish to thank Michael Weiner, Bruce Miller, Howard Rosen, and Helena Chui for access to data.

\section{References}

1. Ashburner, J., Friston, K.: Voxel-based morphometry-The methods. Neuroimage 11 (2000) 805-821

2. Davatzikos, C., Genc, A., Xu, D., Resnick, S.: Voxel-based morphometry using the RAVENS maps: Methods and validation using simulated longitudinal atrophy. Neuroimage 14 (2001) 1361-1369

3. Gaser, C., Nenadic, I., Buchsbaum, B., Hazlett, E., Buchsbaum, M.: Deformationbased morphometry and its relation to conventional volumetry of brain lateral ventricles in MRI. Neuroimage 13 (2001) 1140-1145

4. Pettey, D., Gee, J.: Using a linear diagnostic funcation and non-rigid registration to search for morphological differences between populations: An example involving the male and female corpus callosum. In: Proceedings SPIE Medical Imaging. Volume 4322., SPIE (2001) 1636-1644

5. Jack, C., Petersen, R., O'Brien, P., Tangalos, E.: MR-based hippocampal volumetry in the diagnosis of Alzheimer's disease. Neurology 42 (1992) 183-188

6. Rombouts, S., Barkhof, F., Witter, M., Scheltens, P.: Unbiased whole-brain analysis of gray matter loss in Alzheimer's disease. Neurosci Lett 285 (2000) 231-233

7. Tanabe, J., Amend, D., Schuff, N., DiSclafani, V., Ezekiel, F., Norman, D., Fein, G., Weiner, M.: Tissue segmentation of the brain in Alzheimer disease. Am J Neuroradiol 18 (1997) 115-123

8. Fox, N., Freebourough, P.: Brain atrophy progression measured from registered serial MRI: validation and application to Alzheimer's disease. J Mag Res Imaging 7 (1997) 1069-1075 
9. Jack, C., Petersen, R., Xu, Y., O’Brien, P., Smith, G., Ivnik, R., Boeve, B., Tangalos, E., Kokmen, E.: Rates of hippocampal atrophy correlate with change in clinical status in aging and AD. Neurology 55 (2000) 484-489

10. Cardenas, V., Du, A., Hardin, D., Ezekiel, F., Weber, P., Jagust, W., Chui, H., Schuff, N., Weiner, M.: Comparison of methods for measuring longitudinal brain change in cognitive impairment and dementia. Neurobiology of Aging 24 (2003) $537-544$

11. Fox, N., Scahill, R., Crum, W., Rossor, M.: Correlation between rates of brain atrophy and cognitive decline in AD. Neurology 52 (1999) 1687-1689

12. Chan, D., Janssen, J., Whitwell, J., Watt, H., Jenkins, R., Frost, C., Rossor, M., Fox, N.: Change in rates of cerebral atrophy over time in early-onset Alzheimer's disease: Longitudinal MRI study. Lancet 362 (2003) 1121-1122

13. Smith, C., Malcein, M., Meurer, K., Schmitt, F., Markesbery, W., Pettigrew, L.: MRI temporal lobe measures and neuropsychologic function in Alzheimer's disease. J Neuroimaging 9 (1999) 2-9

14. Worsley, K., Evans, A., Marrett, S., Neelin, P.: A three-dimensional statistical analysis for CBF activation studies in human brain. J Cereb Blood Flow Metab 12 (1992) 1040-1042

15. Friston, K., Holmes, A., Worsley, K., Poline, J., Frith, C., Frackowiak, R.: Statistical parametric maps in functional imaging: A general linear approach. Human Brain Mapping 2 (1995) 189-210

16. Studholme, C., Novotny, E., Zubal, I., Duncan, J.: Estimating tissue deformation between functional images induced by intracranial electrode implantation using anatomical MRI. Neuroimage 13 (2001) 561-576

17. Studholme, C., Constable, R., Duncan, J.: Accurate alignment of functional epi data to anatomical MRI using a physics based distortion model. IEEE Trans Med Imaging 19 (2000) 1115-1127

18. Studholme, C., Cardenas, V., Maudsley, A., Weiner, M.: An intensity consistent filtering approach to the analysis of deformation tensor derived maps of brain shape. Neuroimage 19 (2003) 1638-1649

19. Studholme, C., Cardenas, V., Blumenfeld, R., Schuff, N., Rosen, H., Miller, B., Weiner, M.: Deformation tensor morphometry of semantic dementia with quantitative validation. Neuroimage 21 (2004) 1387-1398

20. Studholme, C., Cardenas, V., Weiner, M.: Building whole brain maps of atrophy rate from multi-subject longitudinal studies using free-form deformations. In: Proceedings of the ISMRM, ISMRM (2001) 